\title{
Transaction
}

\section{Favorable 3D-network Formation of Chitin Nanofibers Dispersed in Water Prepared Using Aqueous Counter Collision}

\author{
Ryota Kose and Tetsuo Kondo \\ Graduate School of Bioresource and Bioenvironmental Sciences, Kyushu University, \\ 6-10-1, Hakozaki, Higashi-ku, Fukuoka 812-8581, Japan
}

\begin{abstract}
In this study, chitin nanofibers were at first successfully prepared using aqueous counter collision (= ACC) method. Dispersion states of the chitin nanofibers in water were compared with aqueous dispersion of cellulose nanofibers in dynamic viscoelastic properties. Morphology of the chitin nanofibers was observed by transmission electron microscopy and polarizing light microscopy. As a result, the ACC treatment provided aqueous dispersion of chitin nanofibers having width of $10-20 \mathrm{~nm}$, and repetition of the treatment allowed a homogeneous aqueous dispersion of the similar sized nanofibers, instead of further pulverization, from the intermediately treated mixture containing different scaled chitin fibers co-existed. The homogeneous dispersion containing chitin nanofibers exhibited much higher dynamic storage and loss moduli when compared with an aqueous cellulose nanofiber system. The dynamic storage modulus of the chitin dispersion tended to increase with increase in the amount of such homogeneous nanofibers, differing from the case of nano-size effects found in the cellulose system that was oppositely decreased. This indicated that the chitin nanofibers dispersed in water were likely to engage a more favorable network.
\end{abstract}

(Received 14 December, 2010 ; Accepted 24 January, 2011)

\section{Introduction}

Chitin is a main component that is located in the outer skins or cuticles of arthropods, and shells in crab and shrimps. Native chitin has a fine hierarchical structure consisting of $\alpha$ - or $\beta$ - chitin nanofibers, various types of proteins and minerals $[1,2]$. The long $N$-acetylaminoglucan chain of a chitin molecule is assembled together to form a fibril with $3 \mathrm{~nm}$ in diameter. The fibrils are wrapped with proteins to be further assembled into a nanofiber of ca. $60 \mathrm{~nm}$ in diameter. The nanofibers are lead up to a bundle with ca. $1 \mu \mathrm{m}$ in diameter, which are then arranged parallel to each other to establish a horizontal plane.

Chitin nanofibers have been attracting a considerable interest in various applications because of the high specific surface areas, high porosity and so on. For examples, scaffolds for tissue engineering [3] have been already proposed. But, there is still limited to a certain area. Therefore, chitin nanofibers, which are believed as a great potential material, have been extensively prepared by several research groups [4-6].

Chitin and cellulose are both structural polysaccharides consisting of $\beta$-(1 $\rightarrow 4)$-linked $N$-acetyl anhydroglucosamine and anhydroglucose units, respectively. These fibers are not soluble in water, but cellulose nanofibers could be at least dispersed in water by aqueous counter collision ( $=$ ACC) method $[7,8]$ that is capable to cleave the interfacial interaction only by water jets without chemical modification of the molecules In the present study, chitin nanofibers were at first attempted to be prepared using ACC method before investigation of the dispersed state of the treated chitin in water in comparison with aqueous dispersion of cellulose nanofibers. In fact, the ACC treatment succeeded to provide nanofibers from native chitin, and thereby it was found that the chitin nanofibers dispersed in water exhibited a favorable network formation when compared with an aqueous cellulose nanofiber (= "nanocellulose") system from microbial pellicle secreted by Gluconacetobacter xylinus [8].

\section{Experimental method}

\subsection{Materials}

$\alpha$-Chitin powder from crab was purchased from Wako Co., Ltd., which was used without further purification. Following reagents were used as components of the culture medium for Gluconacetobacter xylinus [8] : D-Glucose, citric acid and sodium hydroxide with a culture grade were purchased from Wako Chemicals Co., Ltd. Di-Sodium hydrogenphosphate heptahydrate with a 
culture grade was purchased from Nacalai Tesque, Inc. Yeast extract and peptone were provided from Bectom, Dickinson and Co.

\subsection{Preparation of chitin nanofibers dispersed in water}

In ACC system as shown in Fig. 1, ejecting a liquid suspension of the sample from a pair of the nozzles under a high pressure of $200 \mathrm{MPa}$ forms a pair of jets. The diameter of the nozzle was $160 \mu \mathrm{m}$, whereas the angle of collision between the two jets is typically set to be ca.170 degree. The number of ejecting steps (= Pass) as well as the ejecting pressure is adjusted to subject the sample to an appropriate degree of pulverization. Namely, the sample was supposed to be more downsized by repeating the collision with changing the Pass and the desired ejecting pressure [7].

In the present study, the aqueous dispersion with $\alpha$ chitin powder $(0.4 \%(\mathrm{w} / \mathrm{w}))$ was provided for $\mathrm{ACC}$ treatment $[7,8]$ using ACC system (Sugino Co., Japan) under $200 \mathrm{MPa}$ of the ejecting pressure with 1, 5, 10, 30, 60 and 120 Pass, resulting in an aqueous dispersion of chitin nanofibers.

\subsection{Preparation of cellulose nanofibers (= nanocellulose) dispersed in water [8]}

Following inoculated into the SH culture medium [9] in a sterilized plastic container, Gluconacetobacter xylinus (= Acetobacter xylinum: ATCC53582) was cultured statically at $30^{\circ} \mathrm{C}$ to obtain a gel-like membrane having a 3D network structure of the secreted cellulose nanofibers, termed microbial cellulose pellicle. After 2 weeks' incubation, the pellicle with ca. $1 \mathrm{~cm}$ in thickness was established with covering the top of the culture medium. The pellicle was then washed with $0.1 \%$ aqueous $\mathrm{NaOH}$ solution at $80{ }^{\circ} \mathrm{C}$ for 4 hours and successively with water in order to remove protein,

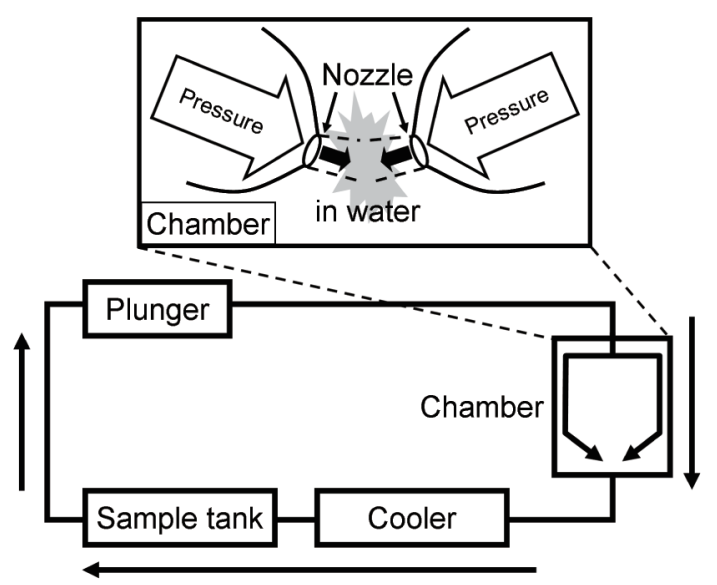

Fig. 1 Aqueous counter collision system using a pair of water jets. bacterial cells and other residues. The purified pellicle was cut into ca. $1 \mathrm{~cm}^{3}$ cubes by a scissors, prior to mix with deionized water. The mixture containing $0.4 \%$ (w/w) cellulose fibers was treated using a homogenizer, Physcotron NS-51 (Microtec Co., Ltd.) for $5 \mathrm{~min}$ at ca. $20,000 \mathrm{rpm}$. The obtained suspension was employed for ACC treatment using ACC system under $200 \mathrm{MPa}$ of the ejecting pressure with 5, 30, 60 and 120 Pass in order to disperse cellulose nanofibers, namely nanocellulose, into water.

\subsection{Transmission electron microscopy (TEM)}

Width of the $\alpha$-chitin and microbial cellulose fibers treated by ACC method was estimated by digitized data from electron micrographs of the sample specimens. For TEM observation of a sample, $0.4 \%(\mathrm{w} / \mathrm{w})$ of the treated fiber dispersions was diluted to $1 / 10$ concentration by deionized water. The mixture was sonicated with an ultrasonic apparatus for 10 seconds, before mounted on a copper grid. Following the excessive was removed using filter paper, the fibers were negatively stained by aqueous 2\% uranyl acetate and finally air-dried. Specimens thus prepared were observed with a JEM-1010 (JEOL Co., Ltd.) operated at $80 \mathrm{kV}$ of the accelerating voltage with a beam current less than $70 \mu \mathrm{A}$. TEM images were acquired at magnifications from $2 \mathrm{k}$ to $50 \mathrm{k}$ in negative films. The images were scanned to be digitized for measurements of width in the individual fiber using Image Pro Plus software v. 4.1 (Media Cybernetics).

\subsection{Polarizing light microscopy}

Polarizing light microscopy was employed for observation of $\alpha$-chitin powders in a micro-size. A drop of $0.4 \%(\mathrm{w} / \mathrm{w})$ of the chitin sample was put on a slide glass, before observation using a polarizing light microscope (OLMPUS Co.).

\subsection{Viscoelastic measurements}

Individual aqueous dispersions with chitin and cellulose nanofibers $(0.4 \%(\mathrm{w} / \mathrm{w}))$ obtained by ACC treatment were provided for dynamic viscoelastic measurements, which were performed with a cone-plate type rheometer, Rheosol-G2000 (UBM, Japan). The radii of the cone and plate were $50 \mathrm{~mm}$ and the angle of the cone was $2^{\circ}$. The rheometer was equipped with a reservoir to prevent the sample drying during the measurements. The measurements were performed at 25 $\pm 0.1{ }^{\circ} \mathrm{C}$ with the measuring frequencies ranging from 0.05 to $56 \mathrm{rad} / \mathrm{s}$. The dynamic strain amplitude $(\gamma)$ was 0.206 . 


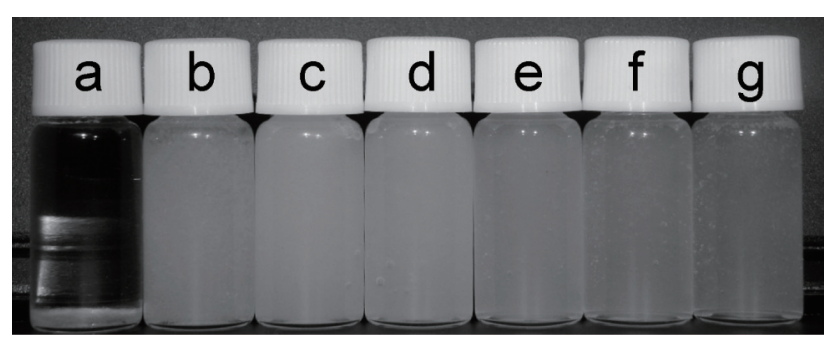

Fig. 2 Photographs of chitin fiber dispersions prepared by ACC treatment with (a) 0 , (b) 1 , (c) 5, (d) 10, (e) 30, (f) 60 and (g) 120 Pass, respectively.

\section{Results and Discussion}

\subsection{Preparation of chitin nanofibers}

The aqueous dispersion containing $\alpha$-chitin powders before ACC treatment was phase-separated (Fig. 2a). With proceeding of the ACC treatment, chitin samples became turbid (Fig. 2b-g). Figs. 3 and 4 show polarizing light microscopic and transmission electron microscopic (TEM) images of chitin samples at each Pass number of the ACC treatment. Chitin particles having micro-sizes in diameter were clearly observed in polarizing light

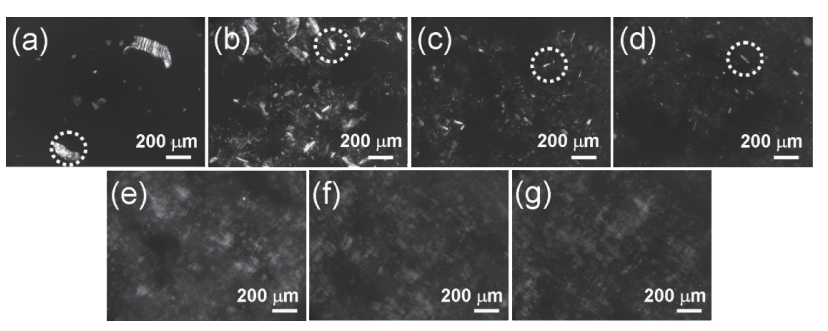

Fig. 3 Polarizing light microscopic images of chitin fiber dispersions prepared by ACC treatment with (a) 0, (b) 1, (c) 5, (d) 10, (e) 30, (f) 60 and (g) 120 Pass, respectively. The substances surrounded by dotted lines in chitin fiber dispersions in the range from 0 to 10 Pass indicate chitin particles with a micro-size.

microscopy, as surrounded by the dotted lines in Fig. 3a-d in the range from 0 to 10 Pass, whereas such particles could not be observed any more after 30 Pass until 120 Pass. At the nanoscale, chitin nanofibers were observed after ACC treatment using TEM (Fig. 4b-g). The amount of chitin nanofibers were apparently increased with increasing in Pass number in the range from 1 to 30 Pass (Fig. 4b-e). Insets in Fig. 4 were enlarged in order to

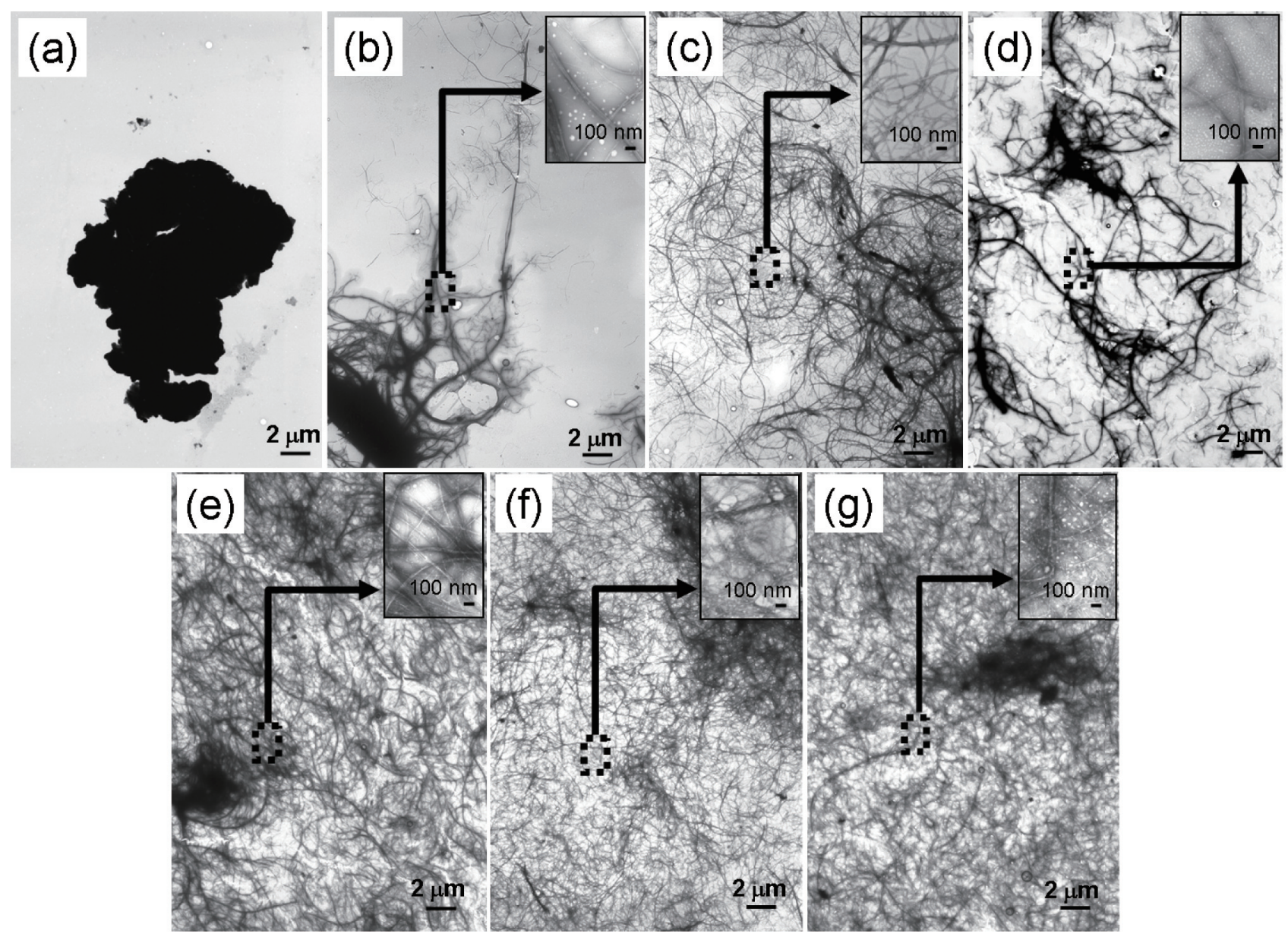

Fig. 4 Transmission electron microscopic images of chitin fiber dispersions prepared by ACC treatment with (a) 0 , (b) 1, (c) 5, (d) 10, (e) 30, (f) 60 and (g) 120 Pass, respectively. Inset in each image is magnified figure to measure the width of the chitin nanofiber. 
Table 1 Width of chitin and cellulose nanofibers depending on Pass number of ACC treatment.

\begin{tabular}{ccc}
\hline Pass number & \multicolumn{2}{c}{ Width / nm } \\
$\alpha$-Chitin & $\begin{array}{c}\text { Microbial } \\
\text { in this study }\end{array}$ & cellulose \\
\hline 0 & - & $64 \pm 40$ \\
1 & $18 \pm 18$ & - \\
5 & $12 \pm 7$ & $43 \pm 33$ \\
10 & $15 \pm 9$ & - \\
30 & $14 \pm 10$ & $23 \pm 10$ \\
60 & $10 \pm 6$ & $22 \pm 16$ \\
120 & $13 \pm 8$ & $20 \pm 13$ \\
\hline
\end{tabular}

measure the fiber width. The results of the width for chitin nanofibers depending on Pass number were listed in Table 1. The width was not significantly changed in the range from 5 to 120 Pass, indicating that once width of the treated chitin nanofibers reached 10-20 nm, the further pulverization did not occurred any more. Instead, the chitin nanofibers in the suspension were homogeneously dispersed in water with increasing in Pass number, by taking the standard deviations into account. On the other hand, the width of nanocellulose decreased rapidly from 60 to $20 \mathrm{~nm}$ with increasing in the Pass number between 0 and 30 Pass. It is indicated that finer chitin nanofibers could be provided easier when compared with nanocellulose.

\subsection{Dispersion states of chitin and cellulose nanofibers in water}

After chitin and cellulose samples obtained by ACC treatment with 60 Pass were kept for 3 months, appearance of the respective samples is shown in Fig. 5. Nanocellulose was still dispersed homogeneously in water, whereas chitin nanofibers were already aggregated in the bottom. This indicates that chitin nanofibers tended to engage a network more preferably than the nanocellulose in water.

Fig. 6 shows a frequency dependence of dynamic storage modulus, $G^{\prime}$ and loss modulus, $G^{\prime \prime}$ of the

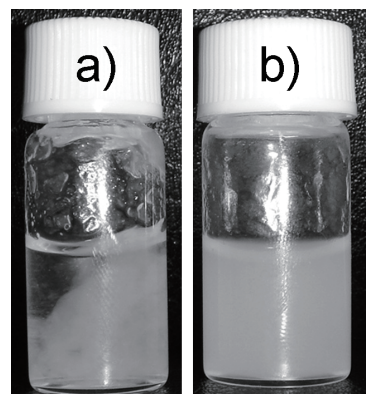

Fig. 5 The appearances of a) chitin and b) cellulose samples by ACC treatment with 60 Pass after keeping for 3 months.

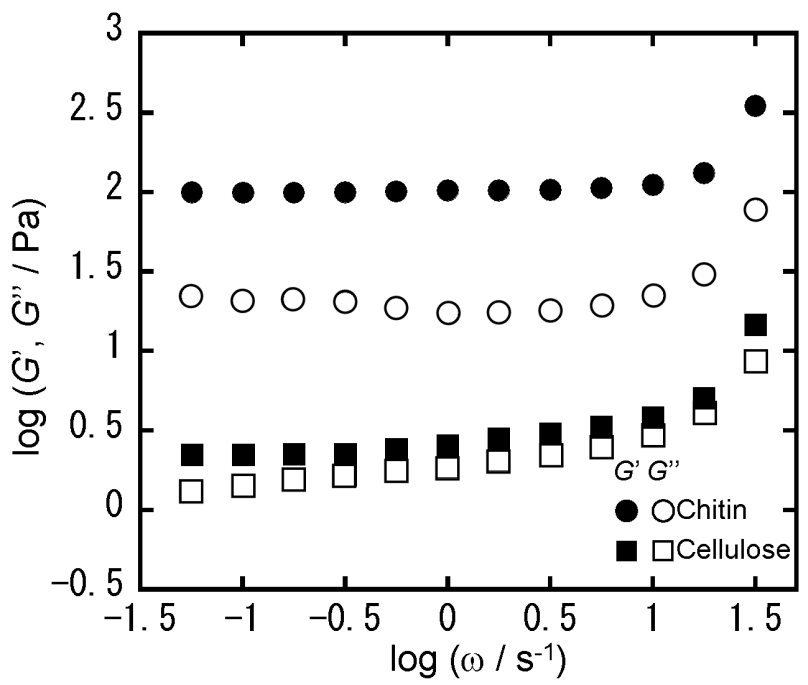

Fig. 6 Dynamic storage modulus, $G^{\prime}$, and loss modulus, $G^{\prime \prime}$, vs. angular frequency, $(\omega)$, for chitin and cellulose nanofiber dispersions treated by the ACC treatment with 30 Pass. Chitin and cellulose concentration was set to be $0.4 \%(\mathrm{w} / \mathrm{w})$.

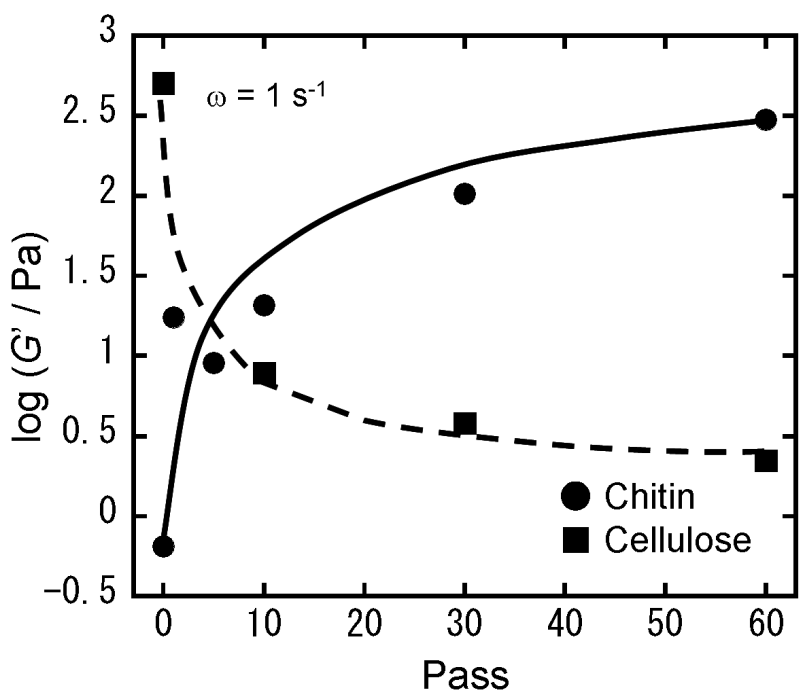

Fig. 7 Change of dynamic storage modulus, $G^{\prime}$, at the angular frequency $(\omega)$ of $1.0 \mathrm{~s}^{-1}$, for chitin and cellulose nanofiber dispersions depending on Pass number of ACC treatment. Chitin and cellulose concentration was set to be $0.4 \%$ $(\mathrm{w} / \mathrm{w})$.

dispersion containing either chitin or cellulose nanofibers obtained by ACC treatment with 30 Pass. Both moduli of the chitin systems exhibited higher values than those in the cellulose system. In addition, the difference in magnitude between $G$ ' and $G$ " of the dispersion containing chitin nanofibers was much larger than that of nanocellulose, independent of the angular frequency $(\omega)$. These results indicated that the chitin dispersion had a higher mechanical strength; namely, the network was engaged strongly to result in a more solid-like structure. 
The modulus $G$ ' was monitored as a function of Pass number, when the angular frequency, $\omega$, was set to be $1.0 \mathrm{~s}^{-1}$, as shown in Fig. 7. The $G$ ' values of the chitin nanofibers increased, but those of nanocellulose decreased with increasing in Pass number. As described above, the amount of chitin nanofibers increased without change of the width as proceeding of Pass number in ACC treatment. On the other hand, although the amount of nanocellulose was also increased, width of the nanofibers decreased oppositely with increasing in the Pass number [8]. Therefore, it indicates that the engagement between the similar sized chitin nanofibers that were more homogeneously dispersed in water might easily provide a three-dimensional aggregation to be phase-separated. It is also possible that the surface of the fibrillated nanocellulose could adsorb a larger amount of water by a "nano-size effect", resulting in a more homogeneous dispersion in water.

\section{Conclusion}

Chitin nanofibers with $10-20 \mathrm{~nm}$ in width were successfully prepared in an aqueous dispersion state from native $\alpha$-chitin using ACC treatment. The large amount of chitin nanofibers with the same range of the nano-size dispersed in water was obtained by changing the treatment conditions. Further, the chitin nanofibers dispersed in water tended to favorably form an aggregation as a 3D-network, whereas nanocellulose, which is a cellulose nanofiber originated from microbial cellulose, was still dispersed homogeneously in water even after a few months.

\section{References}

1. P. -Y. Chen, A. Y. -M. Lin, J. McKittrick, and M. A. Meyers, Acta Biomater., 4, 587 (2008).

2. D. Raabe, C. Sachs, and P. Romano, Acta Materi., 53, 4281 (2005).

3. H. K. Noh, S. W. Lee, J. -M. Kim, J. -E. Oh, K. -H. Kim, C. -P. Chung, S. -C. Choi, W. H. Park, and B. -M. Min, Biomaterials . 27, 3934 (2006).

4. B. -M. Min, S. W. Lee, J. N. Lim, Y. You, T. S. Lee, P. H. Kang, and W. H. Park, Polymer, 45, 7137 (2004).

5. S. Ifuku, M. Nogi, K. Abe, M. Yoshioka, M. Morimoto, H. Saimoto, and H. Yano, Biomacromolecules, 10, 1584 (2009).

6. Y. Fan, T. Saito, and A. Isogai, Biomacromolecules, 9, 1919 (2008).

7. T. Kondo, M. Morita, K. Hayakawa, and Y. Onda, United States Patent 7,357,339 (2005).

8. R. Kose, I. Mitani, W. Kasai, and T. Kondo, Biomacromolecules, in press (DOI : 10.1021/bm 1013469).

9. S. Hestrin and M. Schramm, Biochem. J., 58, 345 (1954). 